Proceedings of the 1994 IEEE International Conference on Robotics and Automation, San Diego, CA, May 9-13, 1994.

\title{
Mapping Tasks into Fault Tolerant Manipulators
}

\author{
Christiaan J.J. Paredis and Pradeep K. Khosla \\ Department of Electrical and Computer Engineering and \\ The Robotics Institute, \\ Carnegie Mellon University, \\ Pittsburgh, PA 15213
}

\begin{abstract}
The application of robots in critical missions in hazardous environments requires the development of reliable or fault tolerant manipulators. In this paper, we define fault tolerance as the ability to continue the performance of a task after immobilization of a joint due to failure. Initially, no joint limits are considered, in which case we prove the existence of fault tolerant manipulators and develop an analysis tool to determine the fault tolerant work space. We also derive design templates for spatial fault tolerant manipulators. When joint limits are introduced, analytic solutions become infeasible but instead a numerical design procedure can be used, as is illustrated through an example.
\end{abstract}

\section{1: Introduction}

Consider the use of manipulators in a nuclear environment where equipment has to be repaired or space has to be searched for radioactive contamination. A manipulator that is deployed for this kind of critical missions must have many desirable attributes. It must be versatile. That is, the manipulator system must be capable of accomplishing a wide variety of tasks and possibly be capable of reconfiguring itself. It must be easily maintainable and cost effective. Ease of maintenance would significantly reduce the downtime and therefore increase the utility of the system. It must also be reliable or fault tolerant. That is, if one of the subsystems were to fail, the reduced system should still be capable of accomplishing its mission objectives. The property of fault tolerance is a must in critical missions that may involve high costs if the mission were to fail due to a failure in the manipulator system. Recently, fault tolerant (or failure tolerant) robotics has been the subject of several publications $[1,11,15,16]$, in which different aspects of the problem are addressed. Visinsky et al. [16] propose a framework to include failure detection in fault tolerant robot systems.
Lewis and Maciejewski [11], on the other hand, discuss the importance of the controller and the redundancy resolution algorithm. In this paper, the focus is on kinematic design of fault tolerant manipulators.

\section{2: Definition of fault tolerance}

To set the stage for our development, we define the following properties of fault tolerant manipulators [1]:

- Fault Tolerant (FT) Manipulator: An n-DOF manipulator that will still be able to meet the task specifications, even if any one or more of its joints fail and are frozen at any arbitrary joint angles.

- $\boldsymbol{k}$-Reduced Order Derivative ( $\boldsymbol{k}$-ROD): When $k$ joints of an $n$-DOF manipulator fail, the effective number of joints is $(n-k)$. The resulting faulty manipulator is called a $k$-reduced order derivative.

- Order of Fault Tolerance: An $n$-DOF manipulator is FT of the $k$-th order, if and only if all possible $k$-reduced order derivatives can still perform the specified task. We call the manipulator $k$-fault tolerant.

- Fault Tolerant Work Space (FTWS): The fault tolerant work space of a $k$-FT manipulator is the set of points reachable by all possible $k$-reduced order derivatives.

These definitions differ from the concept of fault tolerance as proposed by Maciejewski [12]. Instead of attributing the property of fault tolerance to a manipulator, he quantifies a measure of fault tolerance for a manipulator posture and describes a technique to determine the optimal FT posture, based on the singular value decomposition of the Jacobian matrix. If a joint fails in this optimal posture, the resulting reduced order derivative will have maximum possible dexterity. However, a failure at a different angle may make the execution of the task impossible. 
In the rest of this paper, if no specific task is mentioned, it is assumed that the task consists of reaching a nonzero volume of points in the task space, i.e., an $m$-dimensional manifold in the $m$-dimensional task space. A manipulator that satisfies the fault tolerance condition only while reaching a manifold of dimension less than $m$, is considered not to be fault tolerant.

\section{3: Properties of fault tolerant manipulators}

\section{1: Existence}

Suppose that we have an $n$-DOF manipulator, $\mathrm{M}_{n}$, that satisfies all the kinematic requirements of a given task. It has been shown that such a manipulator can be found by using the task based design approach described in [8] or [13]. An obvious way to make this manipulator FT is to design every joint with a redundant actuator. If one of the actuators of the resulting $2 n$-DOF FT manipulator were to fail, the redundant actuator could take over and the manipulator would still be functional. Similarly, a $k$-FT manipulator can be constructed by duplicating every DOF $k$ times, resulting in a $(k+1) n$-DOF manipulator. This same manipulator structure can also be interpreted according to the four level subsumptive architecture for FT manipulators, proposed in [15]. The four levels are:

I) Dual Actuators: extra actuators per joint.

II) Parallel Structures: extra joints per DOF.

III) Redundant Manipulators: extra DOFs per arm.

IV) Multiple Arms: extra arms per system.

According to this classification, the $(k+1) n$-DOF manipulator is an $n$-DOF manipulator with $(k+1)$ actuators for every DOF-redundancy of level I. In the rest of this paper, we focus our attention on fault tolerant manipulators with redundancies of level III.

\section{2: Boundary of the fault tolerant work space}

In this section, we show that a boundary point of the FTWS is a critical value (A critical value is an end-effector position that can be reached in a singular configuration, i.e., that is the image of a critical point [2]).

Consider a $k$-FT planar manipulator, M . A boundary point, $\boldsymbol{p}_{b}$, of the FTWS has to be an element of the boundary of the work space of at least one ROD, $\mathrm{M}^{*}$, obtained by freezing $k$ joints of $\mathrm{M}$. Indeed, if $\boldsymbol{p}_{b}$ were an interior point of the work spaces of all RODs, then it would by definition be an interior point of the FTWS and not a boundary point. The Jacobian of $\mathrm{M}^{*}, J_{M^{*}}$, can be obtained from the Jacobian of $\mathrm{M}, J_{M}$, by deleting the columns corresponding to the frozen DOFs. Because $\boldsymbol{p}_{b}$ is a boundary point of the work space of $\mathrm{M}^{*}$, the Jacobian of

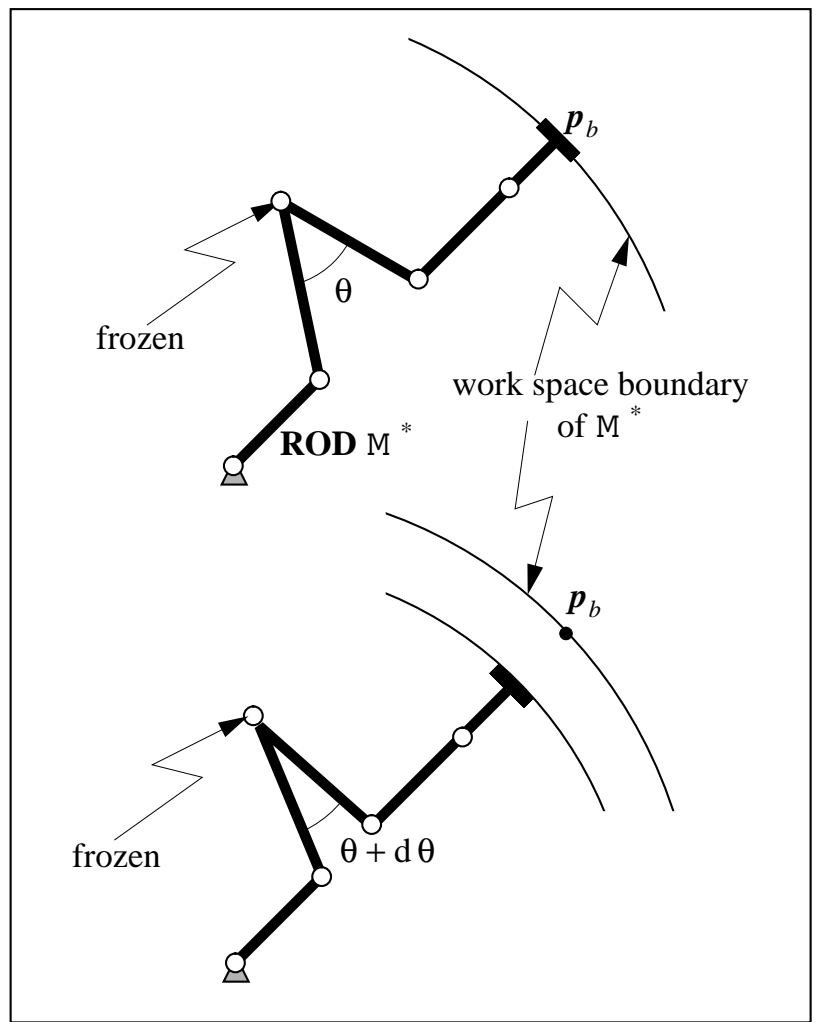

Figure 1: A ROD unable to reach a point outside the FTWS.

$\mathrm{M}^{*}$ at $\boldsymbol{p}_{b}$ is singular. We prove now that $J_{M}$ is singular too. Suppose that $J_{M}$ were non-singular, then at least one of the columns corresponding to a frozen DOF would be outside the column space of the singular matrix, $J_{M^{*}}$. Physically this means that a small change in the angle of that frozen DOF would cause the end effector of $\mathrm{M}$ to move in a direction with a component perpendicular to the boundary of the work space of the ROD, $\mathrm{M}^{*}$, as illustrated in Figure 1. The ROD with this new frozen angle would be unable to reach the point, $\boldsymbol{p}_{b}$. As a result, $\boldsymbol{p}_{b}$ would be outside the FTWS, contradicting the fact that $\boldsymbol{p}_{b}$ is a boundary point of the FTWS. Thus, $J_{M}$ is singular and $\boldsymbol{p}_{b}$ is a critical value.

Consequently, the FTWS is bounded by critical value manifolds. For planar positional manipulators, the critical value manifolds are concentric circles, and the FTWS is an annulus with inner radius $R_{\text {min }}^{\text {FTWS }}$ and outer radius $R_{\max }^{\mathrm{FTWS}}$.

\section{3: Required degree of redundancy}

In Section 3.1, it is shown that, in general, $k n$ redundant DOFs-i.e. $(k+1) n$ DOFs in total-are sufficient to achieve $k$-th order fault tolerance. For planar positional manipulators, we prove now that $2 k$ DOFs are also necessary for $k$-th order fault tolerance. 


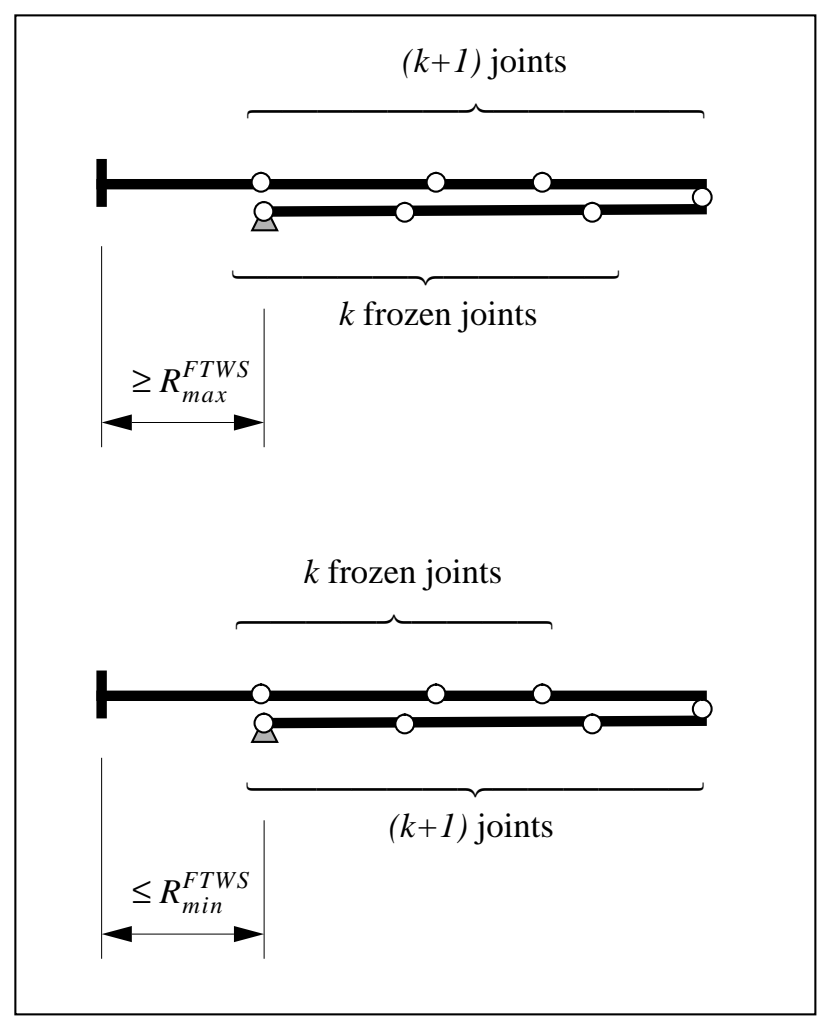

Figure 2: An upper bound for $R_{\max }^{\mathrm{FTWS}}$ and a lower bound for $R_{\min }^{\mathrm{FTWS}}$.

The proof shows that $(2 k+1)$ DOFs (or $2 k-1$ redundant DOFs) are insufficient, by finding a lower bound for $R_{\min }^{\text {FTWS }}$ and an upper bound for $R_{\max }^{\text {FTWS }}$. First consider the ROD obtained by freezing the first $k$ joints at 0 radians, as illustrated in Figure 2. The maximum reach in the opposite direction is an upper bound for $R_{\max }^{\mathrm{FTWS}}$ :

$$
R_{\max }^{\mathrm{FTWS}} \leq-\sum_{i=1}^{k} l_{i}+l_{k+1}+\sum_{i=k+2}^{2 k+1} l_{i}
$$

where $l_{i}$ is the length of the $i$-th link. In order for $R_{\max }^{\mathrm{FTWS}}$ to be positive, we must have that:

$$
\sum_{i=1}^{k} l_{i} \leq l_{k+1}+\sum_{i=k+2}^{2 k+1} l_{i} .
$$

Making this assumption, we find that $R_{\text {min }}^{\text {FTWS }}$ is bounded below by the inner radius of the work space of the ROD obtained by freezing the $k$ last joints at 0 radians, as illustrated in Figure 2:

$$
R_{\min }^{\mathrm{FTWS}} \geq \sum_{i=k+2}^{2 k+1} l_{i}+l_{k+1}-\sum_{i=1}^{k} l_{i} .
$$

From Equation (1) and Equation (3), it follows that at best

$$
R_{\max }^{\mathrm{FTWS}}=R_{\min }^{\mathrm{FTWS}},
$$

resulting in a one-dimensional FTWS. Therefore, a $(2 k+1)$-DOF manipulator cannot be FT

\section{4: Including orientation}

Thus far, we have only considered planar positional manipulators. The results for positional manipulators can be easily extended to the case in which orientation is considered also, by converting the orientational problem into an equivalent positional problem:

An $n$-DOF manipulator, $\mathrm{M}$, is $k$-FT with respect to a set of points, $W=\left\{\left(x_{i}, y_{i}, \varphi_{i}\right)\right\}$, if and only if:

1. the positional manipulator, $\mathrm{M}^{\prime}$, obtained from $\mathrm{M}$ by deleting its last link, $l_{n}$, is $k$-th order fault tolerant with respect to the set of points $W^{\prime}=\left\{\left(x_{i}-l_{n} \cos \varphi_{i}, y_{i}-l_{n} \sin \varphi_{i}\right)\right\}$,

2. $\mathrm{M}^{\prime}$ is $(k-1)-\mathrm{FT}$ while reaching the points in $W^{\prime}$ in any direction.

The positional manipulator, $\mathrm{M}^{\prime}$, needs at least $(2 k+2)$ DOFs to be $k$-FT with respect to $W^{\prime}$; therefore, the manipulator $\mathrm{M}$ needs at least $(2 k+3)$ DOFs. Now, consider a $(2 k+3)$-DOF manipulator with the first $(2 k+2)$ links having length, $l$, and the last link having length zero. It is easy to verify that this manipulator's $k$-th order FTWS is:

$$
W=\left\{(x, y, \varphi) \mid \sqrt{x^{2}+y^{2}} \leq 2 l \text { and } \varphi \in[0,2 \pi)\right\}
$$

Thus, $(2 k+3)$ DOFs are necessary and sufficient for $k$-th order fault tolerance of planar manipulators when orientation is included

This result and the result obtained in Section 3.3 can be summarized in the following theorem:

\section{Theorem:}

For planar manipulators, $2 k$ redundant DOFs are necessary and sufficient for $k$-th order fault tolerance.

\section{4: Spatial fault tolerant manipulators}

For planar FT manipulators, we were able to prove that $2 k$ is the required degree of redundancy. The proof was based on geometric work space analysis. However, geometric analysis becomes too complex for spatial manipulators, especially since we are dealing with redundant manipulators. Therefore, we will demonstrate some properties of spatial FT manipulators using two examples.

As a first example, consider a 5-DOF spatial positional manipulator. Its Denavit-Hartenberg (D-H) parameters are listed in Table 1. This manipulator is first order FT, 


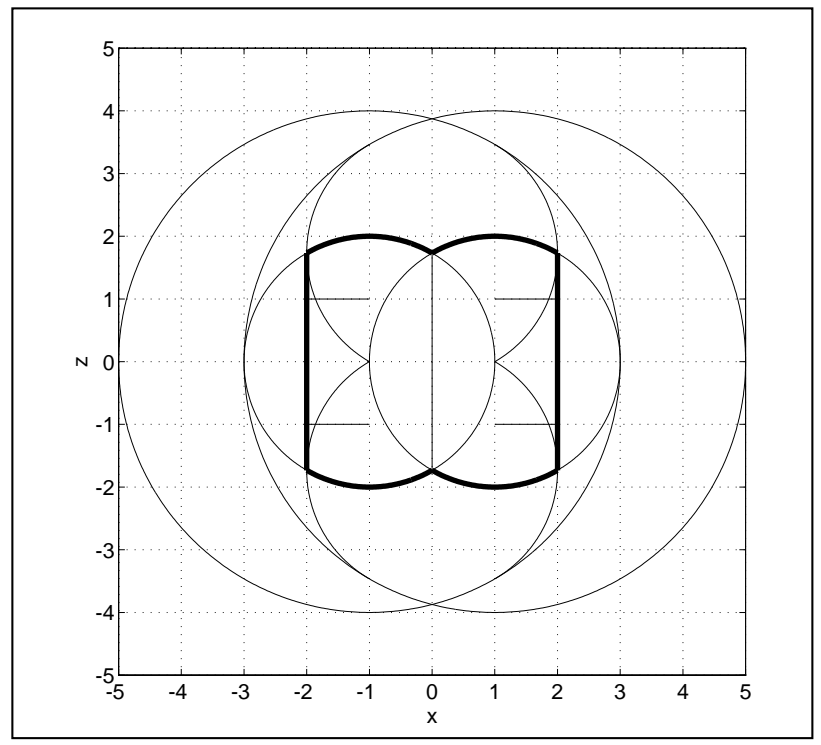

Figure 3: A cross-section of the boundary of the FTWS of a 5-DOF spatial manipulator (bold) as part of its critical value mani-

and because of its simple kinematic structure, an analytic expression for the boundary of the FTWS can be derived. The FTWS is symmetric with respect to the first axis. A cross-section (the $\mathrm{X}-\mathrm{Z}$ plane), as shown in Figure 3, can be described by two segments of a circle with radius 2 and center at $(x=1, z=0)$, and a straight line from $(x=2, z=\sqrt{3})$ to $(x=2, z=-\sqrt{3})$. An important property of this FTWS is that it does not have any holes or a central void, so that the FTWS of the same manipulator scaled by any factor, $\lambda>1$, contains the original FTWS. As a result, this FT manipulator can be used as a design template. Any specified set of points can be reached in a first order FT way by a scaled version of the template.

In Section 3.2, it is shown that the boundary of the FTWS of a planar manipulator coincides with its critical value manifolds. Figure 3 demonstrates that this property also holds for the 5-DOF spatial manipulator considered in this example. The critical value manifolds are comput-

\begin{tabular}{|c||c|c|c|}
\hline DOF $i$ & $d_{i}$ & $a_{i}$ & $\alpha_{i}$ \\
\hline \hline 1 & 0 & 1 & $90^{\circ}$ \\
\hline 2 & $\mathrm{a}$ & 1 & $0^{\circ}$ \\
\hline 3 & $-\mathrm{a}$ & 1 & $90^{\circ}$ \\
\hline 4 & $\mathrm{~b}$ & 1 & $0^{\circ}$ \\
\hline 5 & $-\mathrm{b}$ & 1 & - \\
\hline
\end{tabular}

Table 1: D-H parameters of a 5-DOF first order FT spatial manipulator, without orientation. ed using the algorithm described in [2] and are depicted in a solid line. The bold part of the critical value manifolds is the boundary of the FTWS.

As a second example, consider an 8-DOF manipulator, with D-H parameters listed in Table 2. It is the same manipulator as in example one, with a zero-length 3-rollwrist added at the end. Using a Monte Carlo method, it has been determined that this manipulator is first order FT while reaching all the points in the FTWS of example one, in any direction. This property can be demonstrated with the following arguments. When one of the first five DOFs fails, the manipulator can still reach any position in the FTWS (because the 5-DOF positional manipulator is FT) and can take any orientation at this position using the intact 3-roll-wrist. When one of the DOFs in the wrist fails, we are left with a 7-DOF manipulator which has enough orientational capabilities to reach any point in the FTWS in any orientation. Consequently, one could call this the dextrous FTWS. Since there are again no holes or voids in the FTWS, this manipulator can also be used as a design template.

Finally, one should notice that both examples have only two redundant DOFs, which seems to indicate that the theorem in Section 3.4 is extendable to spatial manipulators.

\section{5: Joint limits}

In Section 2, the definition of a FT manipulator included the specification that joints could fail "at any arbitrary angle." Thus far, we did not consider joint limits and therefore "at any arbitrary joint angle" meant at any angle between 0 and $2 \pi$. From now on, however, an arbitrary joint angle is restricted to be within the joint limits. Using two examples, we discuss now how the introduction of joint limits changes the properties of FT manipulators.

First, consider a 4-DOF positional planar manipulator with links of length one. Figure 4 illustrates how the

\begin{tabular}{|c||c|c|c|}
\hline DOF $i$ & $d_{i}$ & $a_{i}$ & $\alpha_{i}$ \\
\hline \hline 1 & 0 & 1 & $90^{\circ}$ \\
\hline 2 & $\mathrm{a}$ & 1 & $0^{\circ}$ \\
\hline 3 & $-\mathrm{a}$ & 1 & $90^{\circ}$ \\
\hline 4 & $\mathrm{~b}$ & 1 & $0^{\circ}$ \\
\hline 5 & $-\mathrm{b}$ & 0 & $90^{\circ}$ \\
\hline 6 & 1 & 0 & $90^{\circ}$ \\
\hline 7 & 0 & 0 & $90^{\circ}$ \\
\hline 8 & 0 & 0 & - \\
\hline
\end{tabular}

Table 2: D-H parameters of an 8-DOF first order FT spatial manipulator. 


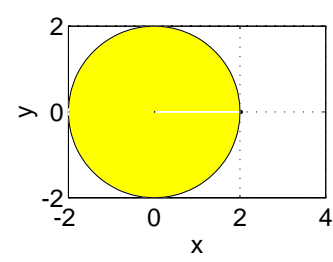

$[-180,180]$

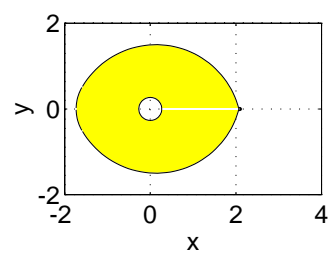

$[-150,150]$

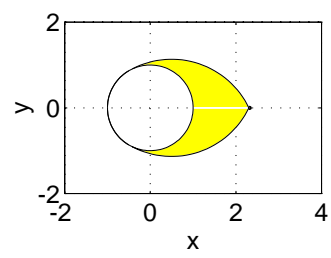

$[-120,120]$

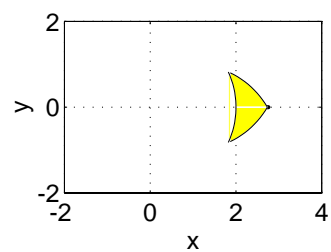

$[-90,90]$

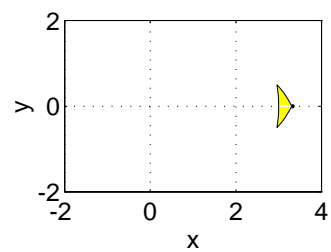

$[-60,60]$

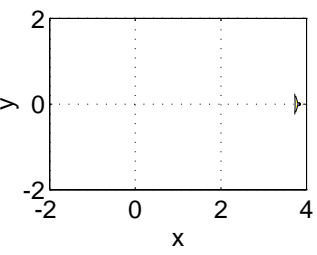

$[-30,30]$

Figure 4: The FTWS of a 4-DOF planar manipulator with joint limits.

work space changes when the joint limits (the same limits for all joints) vary from $\pm 180^{\circ}$ through $\pm 30^{\circ}$. As one would expect the FTWS shrinks, but it also changes position. A point that is outside the FTWS of a manipulator without joint limits can become inside the FTWS when joint limits are introduced. This can be understood by thinking of the FTWS as the intersection of the work spaces of all RODs. When joint limits are introduced, the work space of every ROD reduces in size, but the inter- section of fewer RODs has to be taken to obtain the FTWS.

As a second example, we revisit the 5-DOF spatial manipulator of the previous section. As depicted in Figure 5, the FTWS of this 5-DOF manipulator shrinks rapidly when the joint angle intervals are reduced. Moreover, a central hole appears and the FTWS splits into two disconnected parts. When the joint angle interval becomes smaller than $\left[150^{\circ},-150^{\circ}\right]$, the FTWS vanishes.

From the above examples, it is clear that the analysis, and therefore also the design of FT manipulators is very complex when joint limits are introduced. Whereas we were able to generate design templates for the case of no joint limits (or joint limits of $\pm 180^{\circ}$ ), finding a general design template is infeasible due to the infinite number of possible joint limits. Therefore, in the next section, we propose a numerical procedure to find the kinematic structure of a FT manipulator when joint limits are considered.

\section{6: Task based design of fault tolerant manipu- lators}

\section{1: Mathematical formulation}

In this section, we formulate a method to determine the kinematic structure of a manipulator which is FT with respect to the task of reaching a set of positions/orienta-

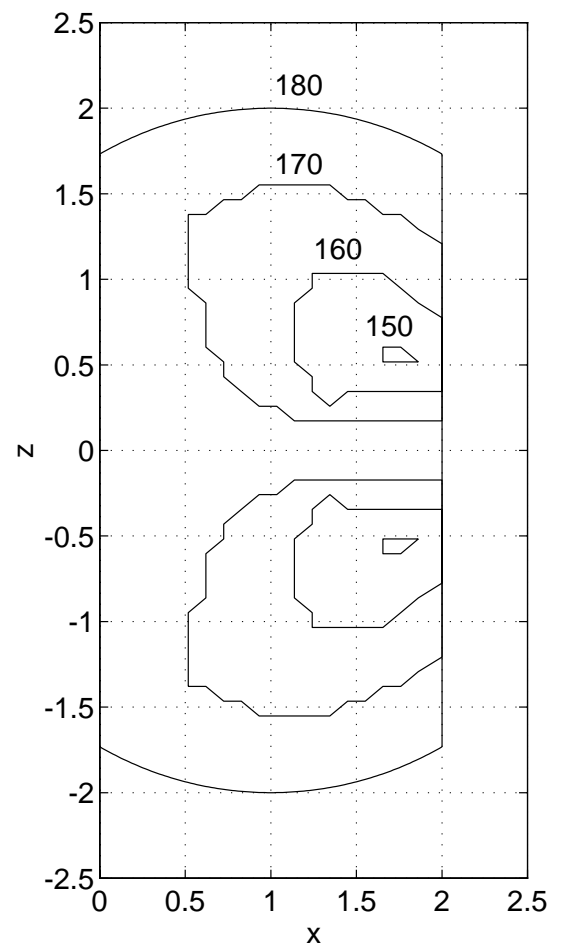

Figure 5: A cross-section of the FTWS of a 5-DOF spatial manipulator with joint limits. 
tions, $W=\left\{\mathbf{p}_{j}=\left(x_{j}, y_{j}, z_{j}, \vartheta_{j}, \varphi_{j}, \psi_{j}\right)\right\}$, and for which the joint limits for each of the DOFs are given. We present a technique based on the design method proposed in [14]. In this approach, the Denavit-Hartenberg parameters are determined through the optimization of a penalty, which is positive when any design specification is violated and is zero when all the specifications are met. This is expressed mathematically as:

$$
\min _{\mathrm{DH}} \sum_{\text {task points }} \min _{\text {postures }}\left[\text { pen_f }\left(\mathrm{DH}, \theta_{\text {post }}, \text { task, cnstr }\right)\right],(6)
$$

where "pen_f $(\cdot)$ " determines the penalty for the violation of the constraints by a manipulator design, $\mathrm{DH}$, in a posture, $\theta_{\text {post }}$, reaching a specific task point.

A manipulator is first order FT with respect to a task if all possible first order RODs can fulfill the task. Equation (5) can then be adapted to include first order fault tolerance as follows [1]:

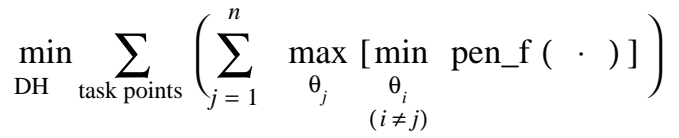

The maximization yields zero when all the RODs, resulting from a failure of joint $j$, are able to reach the task point. As a result, the minimization over the $\mathrm{D}-\mathrm{H}$ parameters equals zero if and only if all possible RODs are able to reach every specified task point.

\section{2: Implementation}

Equation (6) contains three nested optimizations. For each ROD, the innermost minimization finds the posture, for which the penalty function is minimal. The task that we consider is to reach a certain point without violating the joint limit constraints. The penalty function therefore consists of two parts. A first part penalizes the failure to reach the point (without considering joint limits). This penalty is equal to the norm squared of the residue, $\|r\|^{2}$, namely, the distance between the actual position/orientation of the end effector and its desired position/orientation. A second part, penalizes the joint limit violations. This penalty is equal to

$$
\sum_{i=1}^{n}\left\{\max \left(0, \theta_{i}-\theta_{i}^{\max }\right)^{2}+\max \left(0, \theta_{i}^{\min }-\theta_{i}\right)^{2}\right\} .
$$

The resulting minimization of the full penalty function, is a nonlinear least squares (NLLS) problem, and is commonly solved in robotics applications using a NewtonRaphson scheme [6] (cfr. numerical inverse kinematics literature). This scheme converges very quickly when the penalty at the optimum is zero, i.e., for a zero residual NLLS problem. However, it is possible that a task point

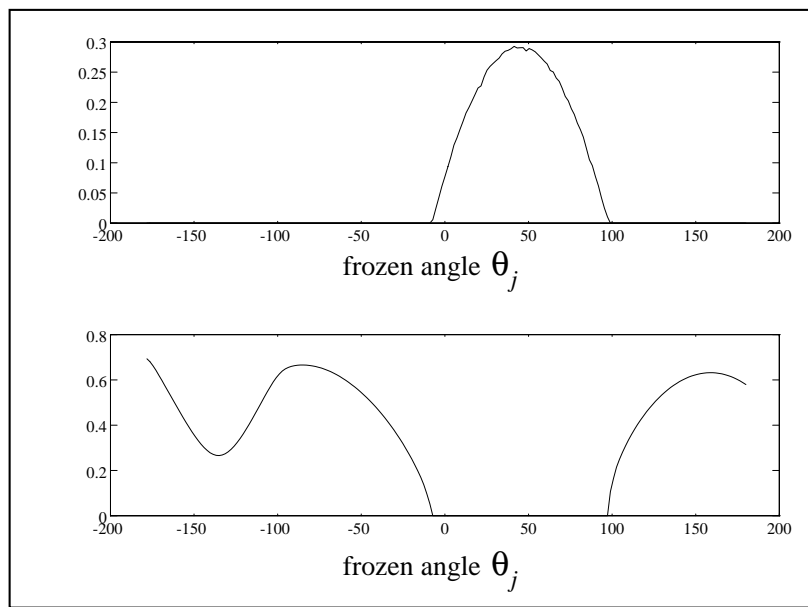

Figure 6: The penalty function and the measure of isotropy.

is outside the reach of the manipulator, in which case the minimization becomes a large residual problem. For this class of problems, the Newton-Raphson scheme (or Gauss-Newton algorithm) converges slowly and is outperformed by the BFGS algorithm (Broyden-Fletcher-Goldfarb-Shanno [5]). As shown by Fletcher and Xu [4], the best overall performance is obtained by a hybrid algorithm, which combines the advantages of both the GaussNewton and BFGS algorithms.

The second optimization in Equation (6) is the maximization over the frozen joint angle, $\theta_{j}$. A typical example of the objective function is shown in Figure 6. Notice that the function and its gradient are zero possibly over a large part of the domain, namely, where the task point is reachable. To solve the maximization, we need to incorporate additional information. The key observation is that when the desired point is outside the reach of the manipulator, the innermost minimization algorithm always gets stuck at the boundary of the work spaces of the manipulator, where the Jacobian matrix of the manipulator loses rank [10]. The measure of isotropy, $\Delta$, is defined as [7]:

$$
\Delta=\frac{\sqrt[m]{\operatorname{det}\left(J J^{T}\right)}}{\operatorname{trace}\left(J J^{T}\right) / m},
$$

with $m$ the number of Cartesian coordinates. For a singular Jacobian, $\Delta$ is zero; otherwise it is positive. Consequently, a minimization over $\Delta$ leads to regions where the penalty is possibly positive. Thus, the derivative of $\Delta$ with respect to $\theta_{j}$, can be used to compensate for the lack of gradient information in the norm of the residue. As one can see in Figure 6, however, the measure of isotropy is a multimodal function of $\theta_{j}$. As there is only one optimization variable, an efficient global optimization algorithm, such as the $P^{*}$ algorithm developed by Zilinskas [17], can be used. $P^{*}$ is a combination of a 


\begin{tabular}{|c|r|r|r|}
\hline \multirow{2}{*}{ DOF $i$} & \multicolumn{3}{|c|}{5 DOF solution } \\
\cline { 2 - 4 } & $d_{i}$ & \multicolumn{1}{|c|}{$a_{i}$} & \multicolumn{1}{c|}{$\alpha_{i}$} \\
\hline \hline 1 & 0.2 & 0.7 & $90^{\circ}$ \\
\hline 2 & 0.1 & 1.0 & $0^{\circ}$ \\
\hline 3 & 0.9 & 0.1 & $135^{\circ}$ \\
\hline 4 & 0.6 & 0.5 & $0^{\circ}$ \\
\hline 5 & 0.1 & 1.3 & - \\
\hline
\end{tabular}

Table 3: D-H parameters of a 5-DOF first order FT spatial solution with joint limits.

one-stage Bayesian algorithm and Brent's local minimization resulting in smart global coverage and accurate local refinement.

The third and outermost optimization minimizes the total penalty of a kinematic configuration, defined by the $(3 n-1)$ D-H parameters. Unfortunately, this function also has multiple minima. Therefore, we need to employ a global optimization method, such as simulated annealing. Simulated annealing was first proposed as a combinatorial optimization algorithm [9], but can be easily adapted to continuously varying optimization variables. The method is basically a random iterative improvement algorithm with the modification that, under certain conditions, an increase in the objective function is accepted. A new trial configuration is generated randomly in the neighborhood of the current configuration. The condition for acceptance of this trial configuration is:

$$
\begin{cases}\Delta F_{o b j} \leq 0 & \Rightarrow \text { accept } \\ \exp \left(-\Delta F_{o b j} / T\right)>\text { random }[0,1) & \Rightarrow \text { accept }\end{cases}
$$

which depends on a control variable, $T$, the temperature. The algorithm is started at a high temperature, for which all new configurations are accepted. After each iteration the temperature is decreased until no new acceptable configuration can be found. The search is then frozen. We adapted this basic algorithm to include the special properties of our objective function, in particular, the algorithm is stopped when a new trial configuration has an objective function value equal to zero, even when the search is not yet frozen. We know that a kinematic configuration with a penalty of zero satisfies all the design requirements.

\section{7: Numerical results}

To illustrate the approach developed in the previous section, we give an example of a first order FT spatial manipulator design: a five DOF spatial manipulator that is required to be first order FT while accomplishing the task of reaching three points:

$$
\begin{aligned}
& \mathbf{p}_{1}=(0.5,0,0.5) \\
& \mathbf{p}_{2}=(0,0.5,0.25) \\
& \mathbf{p}_{3}=(0.75,0,0.5)
\end{aligned}
$$

Notice that we do not consider end effector orientation at this time. The joint limits for all five DOFs are $-150^{\circ}$ and $150^{\circ}$.

From the example in Section 5, we know that five degrees of freedom are sufficient for first order fault tolerance. Our numerical results confirm this. The D-H parameters of a possible design are listed in Table 3. Figure 7 shows a cross-section along the XZ-plane of the boundary of the FTWS.

\section{8: Summary}

In this paper, we defined fault tolerance as a property of a small class of redundant manipulators. Based on the definition, we were able to constructively prove the existence of FT manipulators by duplicating the joints. When no joint limits are considered, we proved analytically that, $2 k$ redundant DOFs are necessary and sufficient for fault tolerance of planar manipulators. We also proved that the boundary of the FTWS consists of critical values. For spatial manipulators, design templates were introduced. A manipulator able to reach a specified set of points can be designed by scaling the template appropriately. For manipulators with joint limits, analytical solutions be-

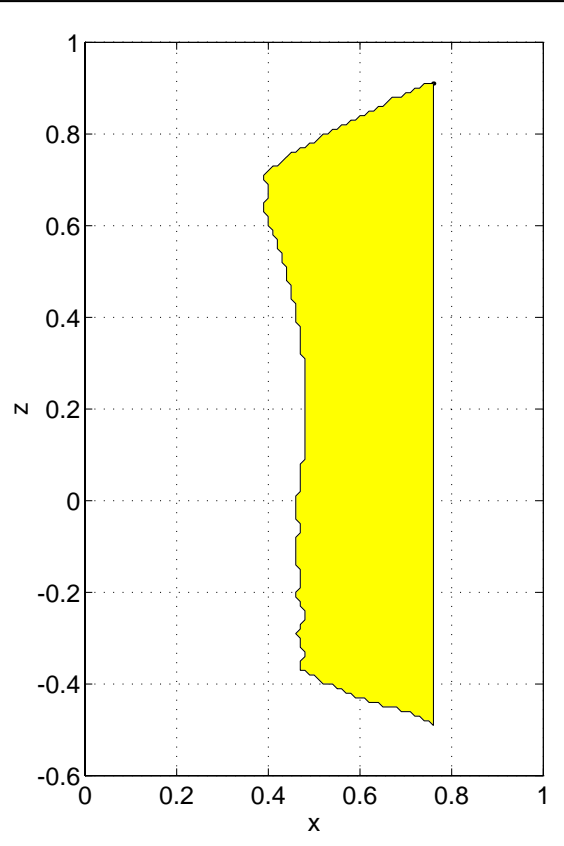

Figure 7: A cross-section of the FTWS of the 5-DOF solution 
come infeasible. Therefore, we introduced a numerical design method based on an earlier developed "task based design" approach. All the different steps of our development were illustrated with design examples.

\section{9: Acknowledgment}

This research was funded in part by DOE under grant DE-F902-89ER14042, by Sandia under contract AC3752-A, by the Department of Electrical and Computer Engineering, and by The Robotics Institute, Carnegie Mellon University.

\section{0: References}

[1] W.K.F. Au, C.J.J. Paredis, P.K. Khosla, "Kinematic Design of Fault Tolerant Manipulators," in Proceedings of the Allerton Conference, October 2, 1992, Urbana-Champagne, Illinois.

[2] J.W. Burdick, "Kinematic Analysis and Design of Redundant Robot Manipulators," Stanford Computer Science Report no. STAN-CS-88-1207, 1988.

[3] J. Denavit, R.S. Hartenberg, "A Kinematic Notation for Lower-Pair Mechanisms Based on Matrices," Journal of Applied Mechanics, Vol. 22, No. 2, pp. 215-221, June 1955.

[4] R. Fletcher, C. Xu, "Hybrid Methods for Nonlinear Least Squares," IMA Journal of Numerical Analysis, Vol. 7, No. 3, pp. 371-389, July 1987.

[5] R. Fletcher, Practical Methods of Optimization, Second Edition, John Wiley \& Sons, New York, 1987.

[6] P.K. Khosla, C.P. Neuman, F.B. Prinz, "An Algorithm for Seam Tracking Applications," The International Journal of Robotics Research, Vol. 4, No. 1, pp. 27-41, Spring 1985.

[7] J.-O. Kim, P.K. Khosla, "Dexterity Measures for Design and Control of Manipulators," in Proceedings of IROS'91:
International Workshop on Intelligent Robots and Systems, pp. 758-763, Osaka, Japan, Nov. 3-5, 1991.

[8] J.-O. Kim, Task Based Kinematic Design of Robot Manipulators, Ph.D. thesis, Robotics Ph.D. Program, Carnegie Mellon University, Pittsburgh, PA, August 1992.

[9] S. Kirkpatrick, C.D. Gelatt Jr., M.P. Vecchi, "Optimization by Simulated Annealing," Science, Vol. 220, No. 4598, pp. 671-680, May 1983.

[10] D. Kohli, M.-S. Hsu, "The Jacobian Analysis of Workspaces of Mechanical Manipulators," Mechanism and Machine Theory, Vol. 22, No. 3, pp. 265-275, 1987.

[11] C.L. Lewis, A.A. Maciejewski, "Dexterity Optimization of Kinematically Redundant Manipulators in the Presence of Joint Failures," to appear in Computers and Electrical Engineering, 1993.

[12] A.A. Maciejewski, "“Fault Tolerant Properties of Kinematically Redundant Manipulators," in Proceedings of the 1990 IEEE International Conference on Robotics and Automation, Cincinnati, Ohio, pp. 638-642, May 1990.

[13] C.J.J. Paredis, An Approach for Mapping Kinematic Task Specifications into a Manipulator Design, M.S. Thesis, Electrical and Computer Engineering Department, Carnegie Mellon University, September 1990.

[14] C.J.J. Paredis, P.K. Khosla, "Kinematic Design of Serial Link Manipulators from Task Specifications," The International Journal of Robotics Research, Vol. 12, No. 3, pp. 274-287, June 1993.

[15] D. Sreevijayan, On the Design of Fault-Tolerant Robotic Manipulator Systems, M.S. Thesis, Mechanical Engineering Department, The University of Texas at Austin, June 1992.

[16] M.L. Visinsky, I.D. Walker, and J.R. Cavallaro, "Layered Dynamic Fault Detection and Tolerance for Robots," in Proceedings of the 1993 IEEE International Conference on Robotics and Automation, Atlanta, Georgia, pp. 180-187, May 1993.

[17] A. Zilinskas, "Optimization of One-Dimensional Multimodal Functions, Algorithm AS 133," Applied Statistics, Vol. 23, pp. 367-375, 1978. 